F. Gross, C.-C. Yang and C. Osgood

Nagoya Math. J.

Vol. 51 (1973), 123-130

\title{
PRIMEABLE ENTIRE FUNCTIONS
}

\author{
FRED GROSS ${ }^{1}$, CHUNG-CHUN YANG ${ }^{2}$ \\ AND CHARLES OSGOOD ${ }^{2}$
}

\section{Introduction.}

An entire function $F(z)=f(g(z))$ is said to have $f(z)$ and $g(z)$ as left and right factors respertively, provided that $f(z)$ is meromorphic and $g(z)$ is entire ( $g$ may be meromorphic when $f$ is rational). $F(z)$ is said to be prime (pseudo-prime) if every factorization of the above form implies that one of the functions $f$ and $g$ is bilinear (a rational function). $\quad F$ is said to be $E$-prime ( $E$-pseudo prime) if every factorization of the above form into entire factors implies that one of the functions $f$ and $g$ is linear (a polynomial). We recall here that an entire nonperiodic function $f$ is prime if and only if it is $E$-prime [5]. This fact will be useful in the sequel.

In this paper we consider the following question: Given an entire function $f(\not \equiv 0)$ can one find an entire function $g$ such that $g(z) f(z)$ is prime? We shall call an entire function $f$ primeable if and only if there exists an entire function $g$ such that $g(z) f(z)$ is prime. We prove that given an entire function $f$ there always exists an entire function $g$ (of zero order) such that $g(z) f(z)$ does not possess any nonlinear polynomial factor. We then apply this result to show that certain classes of entire functions are primeable.

\section{Preliminary Results.}

We shall first prove some theorems which are interesting themselves.

THEOREM 1. Given $F(z)$, an entire transcendental function, there exists an entire function $h(z)$ of order zero such that $h(z) F(z)$ can not

Received May 26, 1972.

1 Mathematics Research Center, Naval Research Laboratory, and University of Maryland, Baltimore County.

2 Mathematics Research Center, Naval Research Laboratory. 
be written as either $p(g)$ or $g(p)$, where $p$ is a polynomial of degree larger than one.

As a preliminary we now show except for a countable set of $a \in C$, $z(z-a) F(z)$ may not be written as $g(p)$, where $p$ is a polynomial of degree larger than one. Suppose the assertion is false. Without loss of generality we may assume that $p(0)=0$ and that $p(z)$ is monic. Then $p(z)=z \prod_{j=1}^{n}\left(z-e_{j}\right)$ for some $n \geq 1$. Since $g(p(0))=0$ we see that the $e_{j}$ are each either equal to $a$ or equal to a zero of $z F(z)$. Then $p(z)=$ $z(z-a)^{\alpha} \prod_{j=1}^{m}\left(z-f_{j}\right)$, for nonnegative integers $\alpha$ and $m$, where each $f_{j}$ is a zero of $z F(z)$. Also, if $\alpha$ is not a root of $z F(z), \alpha$ equals either 0 or 1 , since if $\alpha>1$ it would follow that $z=a$ is a multiple root of $z(z-a) F(z)$. There are at most countably many polynomials $q(z)=$ $z \prod_{j=1}^{m}\left(z-f_{j}\right)$. Since we are assuming the existence of a noncountable number of values of $a$ for which there exists an entire function $g(z)$ with $z(z-a) F(z)=g(p(z))$, one sees that there exist two distinct complex numbers $a_{1}$ and $a_{2}$, neither of which is a zero of $z F(z)$, such that for the same polynomial $q(z)$ and the same choice of $\alpha$ (equal to zero or one)

$$
z\left(z-a_{1}\right) F(z)=g_{1}\left(\left(z-a_{1}\right)^{\alpha} q(z)\right)
$$

and

$$
z\left(z-a_{2}\right) F(z)=g_{2}\left(\left(z-a_{2}\right)^{\alpha} q(z)\right) .
$$

If above $\alpha=0$ we shall show, shortly, that we have a contradiction. If we never have the above situation with $\alpha=0$ we have instead that

$$
z\left(z-a_{j}\right) F(z)=g_{j}\left(\left(z-a_{j}\right) q(z)\right)
$$

for a noncountable set of different $a_{j}$ 's.

If $\alpha=0$ above we have that

$$
\left(z-a_{1}\right)\left(z-a_{2}\right)^{-1}=g_{1}(q(z))\left(g_{2}(q(z))\right)^{-1},
$$

where $q(z)=z \prod_{j=1}^{m}\left(z-f_{j}\right)$. If $m>0$ then, counting multiplicities, we have that should the right hand side above have any poles it has more than one. Since the left hand side has exactly one pole, $m=0$ and we are through in this case.

If we can not assume the above case then consider each $z\left(z-a_{j}\right) F(z)$ $=g_{j}\left(z\left(z-a_{j}\right) q(z)\right)$. Since $g_{j}(0)=0$ we see that

$$
g_{j}\left(z\left(z-a_{j}\right) q(z)\right)=z\left(z-a_{j}\right) q(z) h_{j}\left(z\left(z-a_{j}\right) q(z)\right) .
$$


Also $H(z)=F(z)(q(z))^{-1}$ must be an entire function. We have then that $H(z)=h_{j}\left(z\left(z-a_{j}\right) q(z)\right)$. Let $z_{j}=z_{j}(w)$ denote any solution to the equation

$$
z\left(z-a_{j}\right) q(z)=w .
$$

Then each $H\left(z_{j}(w)\right)=h_{j}(w)$ is entire. Now $z_{j}^{\prime}(w)$ must become infinite when $z_{j}(w)$ equals a zero of $\left(z\left(z-a_{j}\right) q(z)\right)^{\prime}$. Thus, at each zero of $\left(z\left(z-a_{j}\right) q(z)\right)^{\prime}, H^{\prime}(z)$ must be zero. Given any such zero $z_{j}$ we see that

$$
a_{j}=\left(2 z_{j} q\left(z_{j}\right)+z_{j}^{2} q^{\prime}\left(z_{j}\right)\right)\left(q\left(z_{j}\right)+z_{j} q^{\prime}\left(z_{j}\right)\right)^{-1},
$$

unless the denominator vanishes. If there always exists a $z_{j}$, say $z_{j}^{*}$, which leaves the denominator nonzero then we may define a one to one mapping from $a_{j}$ to $z_{j}^{*}$. It would follow that $H^{\prime}(z)$ equals zero on a noncountable set, so $H(z)$ is a constant and $F(z)$ is a polynomial contrary to assumption. This would conclude our present proof. If the denominator vanishes then the denominator, $2 z_{j} q\left(z_{j}\right)+z_{j}^{2} q^{\prime}\left(z_{j}\right)$, must vanish also. Thus, $z_{j} q\left(z_{j}\right)$ vanishes. If $z_{j}=0$ is put in the equation $\left(z\left(z-a_{j}\right) q(z)\right)^{\prime}$ $=0$ we see that $q(0)=0$. Therefore $z_{j}$ must be a zero of $q(z)=0$. This equation is of degree one less than $\left(z\left(z-a_{j}\right) q(z)\right)^{\prime}=0$; hence, there exists a point $z_{j}^{*}$. This proves that $z(z-\alpha) F(z)=g(p)$ is possible for at worst a countable number of different values of $a$.

What we wish to do next is to construct a transcendental function $h_{1}(z)$ of order zero such that $h_{1}(z) f(z)$ can not be written as $p(g)$ where $p$ is a polynomial of degree larger than 1 . We shall see that our $h_{1}(z)$ is such that the same assertion holds for each $(z-a) z h_{1}(z) F(z)$; therefore, we may choose $a$ such that $(z-a) z h_{1}(z) F(z)$ may not be written as $g(p)$ either.

We wish to rely in what follows on the following result essentially due to Borel [2].

LEMma. Suppose that $A(r)>0$ is monotone increasing and $B(r)>0$ is monotone decreasing for all $r \geq r_{0}, A(r)$ and $B(r)$ are continuous, and that $\int_{r_{0}}^{\infty} B(r) d r<\infty$. Then $A(r+B(A(r)))<A(r)+1$ except for, at most, a set of finite measure.

Let $M(r)=\max _{|z| \leq r}|F(z)|$. Then $M(r)>0$ and is monotone increasing if $r \geq 1$. Set $n(r)=(\log (M(r)))^{2 / 3}$. Set $f(r)=\log (M(r+1 / n(r)))$. Then we have

Lemma 1. For any positive constant $N, M(r) / e^{n(r)} f(r) r>N$ for all 
$r$ except, at most, a set of finite measure.

Proof. In the lemma of Borel's set $A(r)=(\log M(r))^{1 / 3}$ and $B(r)=$ $r^{-2}$. Then

$$
f(r)^{1 / 3}=\left(\log \left(M\left(r+(n(r))^{-1}\right)\right)\right)^{1 / 3}<(1+\log (M(r)))^{1 / 3}, \text { so }
$$

$f(r)<2 \log (M(r))$, except on a set of finite measure. The result now follows trivially, since $M(r) \rightarrow \infty$, as $r \rightarrow \infty$, faster than $r^{2}$ say.

LEMMA 2. Except for a set of finite measure

$$
M\left(r+\frac{1}{f(r)}\right)<e^{n(r)} M(r) .
$$

Proof. This is equivalent to $\log (M(r+1 / f(r)))<n(r)+\log (M(r))$ except for a set of finite measure. Since $f(r)>\log (M(r))$ this would be implied by

$$
\log \left(M\left(r+(\log (M(r)))^{-1}\right)\right)<n(r)+\log (M(r)),
$$

except for a set of finite measure. Let us drop references to the exceptional set in what follows. Setting $A(r)=(\log (M(r)))^{1 / 2}$ and $B(r)=$ $r^{-2}$ we see by the Borel's Lemma that $\log \left(M\left(r+(\log (M(r)))^{-1}\right)\right)<((\log$ $\left.(M(r)))^{1 / 2}+1\right)^{2}$. Now $\log M(r)+2(\log M(r))^{1 / 2}+1<n(r)+\log (M(r))$. This proves Lemma 2 .

LEMMA 3. Given $F(z)$ as above and any positive integer $N>0$, we may choose a point $a_{1}$, with $\left|a_{1}\right|=r$, such that $|F(z)|>N$ and $\left\{\left(1-z a_{1}^{-1}\right) F(z) \mid>N\right.$ if $\left(16 e^{n(r)} f(r)\right)^{-1}<\left|z-a_{1}\right|<\left(8 e^{n(r)} f(r)\right)^{-1}$.

Proof: Let $r>0$ be chosen so that the inequalities of Lemmas 1 and 2 are both satisfied (with $2^{5} N$ replacing $N$ in Lemma 1). Let $c_{0}$, $c_{1}$, and $c_{2}$ be circles with center at $a_{1}$ of respective radii, $\left(8 e^{n(r)} f(r)\right)^{-1}$, $(2 f(r))^{-1}$, and $(f(r))^{-1}$. Now if $w$ is inside of $c_{1}$ we see that

$$
\left|F^{\prime}(w)\right|=\left|(2 \pi i)^{-1} \int_{c_{2}} F(z)(z-w)^{-2} d z\right| \leq 4 f(r) e^{n(r)} M(r) .
$$

'Thus if $w$ is inside of $c_{0}$ we see that

$$
|F(w)| \geq M(r)-\left(8 e^{n(r)} f(r)\right)^{-1}\left(4 f(r) e^{n(r)} M(r)\right) \geq \frac{1}{2} M(r)>N .
$$

Then if

$$
\left(16 e^{n(r)} f(r)\right)^{-1}<\left|z-a_{1}\right|<\left(8 e^{n(r)} f(r)\right)^{-1}
$$


we have that

$$
\left|\left(1-\frac{z}{a_{1}}\right) F(z)\right| \geq \frac{\left|z-a_{1}\right|}{\left|2 a_{1}\right|} M(|z|) \geq \frac{M(r)}{2^{5} r f(r) e^{n(r)}}>N
$$

by Lemma 2. This proves Lemma 3 .

LEMMA 4. Let $c$ be a circular path which winds once about $z=a_{1}$ in the positive direction and has radius $3.2^{-5}\left(e^{n(r)} f(r)\right)^{-1}$. Then, under the conditions of Lemma 3 , where $H=\left(1-z / a_{1}\right) F(z)$ we see that $\Gamma=H(c)$ winds once about zero in the positive direction and always stays outside of $|w|<N$.

Proof. The second assertion is trivial. To see the first statement look at

$$
\begin{aligned}
\frac{1}{2 \pi i} \int_{\Gamma} \frac{d w}{w} & =\frac{1}{2 \pi i} \int_{c} \frac{H^{\prime}}{H} d z=\frac{1}{2 \pi i} \int_{c} \frac{d}{d z}\left\{\ln F(z)+\ln \left(1-\frac{z}{a_{1}}\right)\right\} d z \\
& =\frac{1}{2 \pi i} \int_{c} \frac{d}{d z}\left\{\ln \left(1-\frac{z}{a_{1}}\right)\right\} d z=1
\end{aligned}
$$

(since $|F(z)| \geq N$ on and inside of $c$ ). This proves Lemma 4 .

LEMMA 5. If $H_{1}=E(z) H(z)$ where $|E(z)|>\frac{1}{2}$ on and inside of $c$ then Lemma 4 holds with $H_{1}$ substituted for $H$ and $\frac{1}{2} N$ substituted for N.

Proof. Trivial.

Now let us prove our Theorem. Choose two sequences of positive integers $b_{1}, \cdots$, and $c_{1}, \cdots$ such that $\prod_{j=1}^{\infty}\left(1-z / b_{j}\right)$ has zero order of growth and $\prod_{j=1}^{\infty}\left(1-1 / c_{j}\right)>\frac{1}{2}$. Pick $a_{1}$ as in Lemma 3 for $F_{1}(z)=F(z)$ and $N=1$, subject to the conditions that $\left|a_{1}\right|>b_{1}$ and the radius of $c$ is less than one. Proceeding by induction apply Lemma 3 with our $M(r)$ being for the function

$$
F_{n}(z)=\prod_{j=1}^{n-1}\left(1-\frac{z}{a_{j}}\right) F(z) \text { and with } \quad N=n,
$$

subject to the conditions that

$$
\left|a_{n}\right|>b_{n}, \quad \text { each } \quad\left|1-\frac{\left|a_{j}\right|+1}{\left|a_{n}\right|}\right|>\left(1-\frac{1}{c_{n-j}}\right)
$$

for every $1 \leq j \leq n-1$, and the curve $c$ has radius less than one. 
Now set $h_{1}(z)=\prod_{j=1}^{\infty}\left(1-z / a_{j}\right)$. We see that $h(z)$ has order zero. Also each

$$
\left|h_{1}(z) F(z) / F_{n+1}(z)\right|>\frac{1}{2},
$$

if $z$ is on or inside of the curve $c$ about $a_{n}$. Thus by Lemma 5 each curve $\Gamma_{j}=h_{1}(c) F(c)$, where $c$ is about $a_{j}$, winds once about $w=0$ in the positive direction and lies entirely inside of $\{w|| w \mid \geq j / 2\}$.

Suppose now that

$$
h_{1}(z) F(z)=P(g(z))
$$

where $P$ is a polynomial of degree $n>1$. Then for all sufficiently large $j$ we have that on the different curves $c$,

$$
g(z)=\rho^{k}\left(h_{1}(z) F(z)\right)^{1 / n}+K+o\left(\left|h_{1}(z) F(z)\right|^{-1 / n}\right)
$$

for some complex constant $K$ and some positive integer $k$, where $\rho$ is a primitive $n$-th root of unity. Continuing $g(z)$ about $c$ we obtain

$$
\rho^{k+1}\left(h_{1}(z) F(z)\right)^{1 / n}+K+o\left(\left|h_{1}(z) F(z)\right|^{-1 / n}\right) .
$$

If $j$ is chosen sufficiently large we see that the two values must be distinct. Thus $g(z)$ is not entire. This proves that $h_{1}(z) F(z)=p(g)$ is impossible and completes the proof of the Theorem.

THEOREM 2. One may construct $h(z)$ as in Theorem 1 with all of the zeros of $h(z)$ lying asymptotically on a ray $\arg z=\theta_{0}$, unless for each positive integer $n$ there exists an open sector containing $\arg z=\theta_{0}$ on which $z^{n} F(z)$ is bounded.

Proof. Using $z^{n+2} F(z)$ for $F(z)$, where $z^{n} F(z)$ is not bounded on any open sector containing $\arg z=\theta_{0}$, we may go through the above proof using in the $n$-th step $M(r) \stackrel{\text { def. }}{\equiv} \max _{\theta}\left\{\mid F_{n}\left(r e^{i \theta}\right) \| \theta_{0}-1 / n<\theta<\theta_{0}+1 / n\right\}$ instead of $M(r)$ for $F_{n}(z)$. The only properties of $M(r)$ which were needed in the proofs of Lemmas 1 and 2 were monotonicity and (if $r$ was sufficiently large) that $M(r) \geq r^{2}$. This proves Theorem 2 .

\section{Primeable Functions and Main Results.}

THEOREM 3. Let $f$ be a transcendental entire function with $\delta(0$, $f-h)=1$ for some entire function $h(z)$ of order less than that of $f^{\prime} s$. Then $f$ is primeable. 
EXAMPLE. Let $p_{1}, p_{2}, p_{3}$ be polynomials with $p_{2} \not \equiv$ constant. $p_{1} \neq \equiv 0$. Then $p_{1} e^{p_{2}}+p_{3}$ is primeable.

Proof of the Theorem. First of all, we note that under the hypotheses of the theorem $f$ is pseudo-prime. This is an extension of a result of Goldstein's [4], and its proof can be obtained by adopting the argument used in [6]. Thus, if $\delta(0, f-h)=1$ for some entire function $h$ of order less than that of $f$, we have, for any entire function $g(\not \equiv 0)$ of zero order, $\delta(0, g f-g h)=1$. Therefore, $g f$ is also pseudo-prime. Now choose $g$ as in Theorem 1 such that $g f$ is not a periodic function and can not be expressed as $k(q)$ or $q(k)$ where $k$ is transcendental and $q$ is a polynomial of degree at least two. Thus we have shown $g f$ is $E$-prime and therefore is prime.

Along similar lines we have the following result.

THEOREM 4. Let $p$ be a nonconstant polynomial, and $f$ be an entire function of finite order with $\delta(0, f(z)-a(z))=1$ for some entire function $a(z)$ of zero order. Then $p(f)$ is primeable.

THEOREM 5. Let $F$ be an entire function of order $\rho$. Assume that $\rho$ is finite and $\neq \frac{1}{2}$. Suppose there exists a real number $\alpha$ such that for any $\delta>0$, all but a finite number of zeros of $F$ lie in the angle $|\arg z-\alpha|<\delta$, then $F$ is primeable.

Proof. We deal the case $p>\frac{1}{2}$ first. According to a result of Baker's [1] $F$ is pseudo-prime and so is $q(z) F(z)$ for any polynomial $q(z)(\not \equiv 0)$. Now as shown in the previous section that one can always find a $a \in C$ such that $F(a) \neq 0, a \neq 0$ and $z(z-a) F(z)$ may not be written as $g(p)$ where $p$ is a polynomial of degree larger than one. Now suppose that $z(z-a) F(z)=p(g)$ and $P(z)$ has two distinct roots. Then the order of $g$ is at most $1 / 2$ (for the proof we refer the reader to [3]). Therefore the order of $F$ will be $\leq \frac{1}{2}$, giving a contradiction. Then $p$ has to be a monomial. But $a$ is a simple zero of $z(z-a) F(z)$ and hence $p$ has to be a linear function. It follows that $z(z-a) F(z)$ is prime.

The case when $p<\frac{1}{2}$ can be proved by combining Theorem 2 and the preceding argument. Thus the theorem is proved. 


\section{REFERENCES}

[1] I. N. Baker, "The value distribution of composite entire functions", Acta Math. Szeged, Tom 32, (1971).

[ 2 ] E. Borel, "Sur les zéros des fonctions entières", Acta Math., 20, (1897) 357-396.

[ 3 ] A. Edrei, "Meromorphic functions with three radically distributed values", Trans. Amer. Math. Soc., 78, (1955) 271-293.

[4] R. Goldstein, "On factorization of certain entire functions", J. Lond. Math. Soc., (2), (1970) pp. 221-224.

[5] F. Gross, "Factorization of entire functions which are periodic mod g", Indian J. of pure and applied Math., Vol. 2, No. 3, (1971).

[6] F. Gross and C. C. Yang, "The fix-points and factorization of meromorphic functions", Trans. Amer. Math. Soc., Vol. 168, (1972). 